\title{
Stetigkeitswürfel: Spielerisch den Umgang mit der Definition der Stetigkeit erlernen
}

\author{
Oliver C. Schnürer und Jan-Hendrik Treude
}

\begin{abstract}
Die Stetigkeitswürfel sind ein mathematisches Spielzeug, mit dem wir in einem zusätzlichen Kurs für Studienanfänger im Kontext der Definition der $\epsilon$ - $\delta$-Stetigkeit formales Arbeiten geübt haben. Mit unserem Artikel möchten wir diese Würfel bekannt machen und dazu einladen, sie auch andernorts einzusetzen. Auch erfahrene Mathematikerinnen und Mathematiker können durch die Würfel vor kleine Herausforderungen gestellt werden.
\end{abstract}

Der Einstieg in ein Mathematikstudium fällt bekanntlich oft schwer und erfordert neben dem Erlernen neuer mathematischer Inhalte auch das Erlernen einer für die meisten Studienanfänger größtenteils unbekannten Methodik, nämlich den Umgang mit formalen Begriffsdefinitionen und Argumentationen bzw. Beweisen. Um Studienanfänger hierbei gezielt zu unterstützen, findet an der Universität Konstanz seit dem Wintersemester 2016/17 eine neue freiwillige zweisemestrige Lehrveranstaltung mit dem Titel „Einführung in das mathematische Arbeiten (EmA)“ statt (hierzu siehe [1]).

Ein Begriff, der erfahrungsgemäß vielen Studienanfängern Schwierigkeiten bereitet, ist die $\varepsilon$ - $\delta$-Definition der Stetigkeit einer Funktion $f: \mathbb{R} \rightarrow \mathbb{R}$, welche lautet:

$$
\begin{aligned}
\forall x \in \mathbb{R}: \forall \varepsilon>0: \exists \delta>0: \forall y & \in \mathbb{R}: \\
& |x-y|<\delta \Longrightarrow|f(x)-f(y)|<\varepsilon .
\end{aligned}
$$

Genau nachzuvollziehen, wie dieses logische Konstrukt den anschaulich so einfachen Begriff der Stetigkeit erfasst und wie man korrekt mit (1) umgeht, bereitet im ersten Semester häufig schon in konkreten Beispielen Schwierigkeiten. Da wir es wichtig finden, bereits früh im Studium Gewandtheit im Umgang mit diesem und ähnlichen in der Analysis allgegenwärtigen Begriffen zu erlangen, haben wir im Rahmen der EmA die Stetigkeitswürfel entwickelt. Diese ermöglichen einen spielerischen Zugang. Sie lassen sich leicht mit normalen Würfeln und diesem Artikel imitieren.

\section{Beschreibung der Stetigkeitswürfel}

Für die Stetigkeitswürfel haben wir zunächst die Bedingung (1) der Stetigkeit einer Funktion $f: \mathbb{R} \rightarrow \mathbb{R}$ in drei Teile zerlegt. Für jeden der drei Teile haben wir uns anschließend fünf unterschiedliche Variationen ausgedacht, die jeweils die Beschriftungen der Seiten eines Würfels bilden, wobei die ursprüngliche Variante aus der Stetigkeitsdefinition stets doppelt enthalten ist:

$$
\begin{array}{rll}
\varepsilon-\delta \text {-Würfel } & \\
\bullet & \forall \varepsilon>0 & \exists \delta>0 \\
\bullet & \forall \varepsilon>0 & \exists \delta>0 \\
\because & \forall \delta>0 & \exists \varepsilon>0 \\
\because & \exists \varepsilon>0 & \forall \delta>0 \\
\because \because & \forall \varepsilon>0 & \forall \delta>0 \\
\hdashline: & \exists \varepsilon>0 & \exists \delta>0
\end{array}
$$

$$
\begin{array}{ccccc}
x \text {-y-Würfel } & & & \\
\bullet & \forall x \in \mathbb{R} & \ldots & \ldots & \forall y \in \mathbb{R} \\
\because & \forall x \in \mathbb{R} & \ldots & \ldots & \forall y \in \mathbb{R} \\
\hdashline & \ldots & \ldots & \exists x \in \mathbb{R} & \forall y \in \mathbb{R} \\
\vdots & \ldots & \ldots & \forall x \in \mathbb{R} & \forall y \in \mathbb{R} \\
\because & \ldots & \forall x \in \mathbb{R} & \ldots & \forall y \in \mathbb{R} \\
\vdots & \exists x \in \mathbb{R} & \ldots & \ldots & \forall y \in \mathbb{R}
\end{array}
$$

Implikationswürfel

$$
\begin{aligned}
\bullet & |x-y|<\delta & \Longrightarrow|f(x)-f(y)|<\varepsilon \\
\bullet & |x-y|<\delta & \Longrightarrow|f(x)-f(y)|<\varepsilon \\
\because \bullet & |f(x)-f(y)|<\delta & \Longrightarrow|x-y|<\varepsilon \\
\because & |x-y|+\frac{1}{1+|x|}<\delta & \Longrightarrow|f(x)-f(y)|<\varepsilon \\
\because \because & |x+y|<\delta & \Longrightarrow|f(x)-f(y)|<\varepsilon \\
\hdashline & \min \{|x|,|y|\}>\delta & \Longrightarrow|f(x)-f(y)|<\varepsilon
\end{aligned}
$$

Mit dieser Tabelle und handelsüblichen Würfeln lassen sich die Stetigkeitswürfel auch ohne Basteln einfach imitieren.

Der Einsatz der Stetigkeitswürfel funktioniert nun so: Man würfelt jeden der drei Würfel und setzt die gezeigten
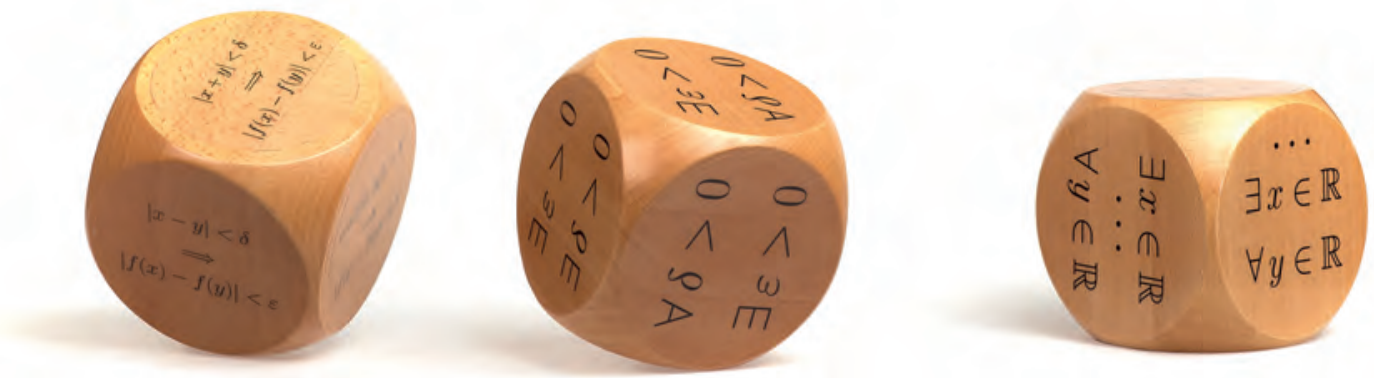
Seiten so zusammen, dass die erwürfelten Ausdrücke des $\varepsilon$ - $\delta$-Würfels in die beiden Leerstellen des $x$ - $y$-Würfels platziert werden und der Implikationswürfel zuletzt kommt. Beispielhaft stelle man sich vor, dass das Würfeln zu folgendem Ergebnis geführt hat:

○ Der $\varepsilon$ - $\delta$-Würfel zeigt

$$
\because: \quad \exists \varepsilon>0 \quad \forall \delta>0
$$

○ Der $x$-y-Würfel zeigt

$$
\because \because \quad \ldots \quad \forall x \in \mathbb{R} \quad \ldots \quad \forall y \in \mathbb{R}
$$

○ Der Implikationswürfel zeigt

$$
\bullet:|x-x|<\delta \Longrightarrow|f(x)-f(y)|<\varepsilon
$$

Hieraus setzt man nun die folgende Aussage zusammen:

$$
\begin{aligned}
\exists \varepsilon>0: \forall x \in \mathbb{R}: \forall \delta>0: \forall y \in \mathbb{R}: \\
\qquad|x-y|<\delta \Longrightarrow|f(x)-f(y)|<\varepsilon .
\end{aligned}
$$

Mit den Würfeln können so 125 Aussagen über eine Funktion $f: \mathbb{R} \rightarrow \mathbb{R}$ erzeugt und so ohne große Mühe immer wieder neue Übungssituationen geschaffen werden.

\section{Ein Beispiel für den Einsatz in einer Vorlesung}

Anhand von (2) wollen wir nun exemplarisch erklären, wie wir die Würfel in einer Vorlesung oder Übung eingesetzt haben. Allgemein besteht das Ziel natürlich darin, die Aussage (2) zu verstehen. Dem kann man sich mittels unterschiedlicher Fragen nähern, wobei es stets zwei prinzipiell verschiedene Sichtweisen gibt: Einerseits kann man überlegen, unter welchen Voraussetzungen an eine Funktion $f: \mathbb{R} \rightarrow \mathbb{R}$ die Eigenschaft (2) zutrifft. Hierbei würde man (2) als eine zu beweisende Aussage behandeln. Andererseits kann man überlegen, welche Folgerungen sich für eine Funktion $f: \mathbb{R} \rightarrow \mathbb{R}$ ergeben, die (2) erfüllt. Hierbei würde man (2) als eine Voraussetzung behandeln. Diese beiden Sichtweisen entsprechen natürlich den Fragen nach hinreichenden bzw. notwendingen Bedingungen für (2).

- Welche Funktionen erfüllen (2) bzw. erfüllen (2) nicht?

Die Studierenden prüfen graphisch oder formal, ob (2) für konkrete Funktionen erfüllt ist. Hierbei wird (2) natürlich als zu beweisende oder zu widerlegende Aussage angesehen. In einem weiteren Schritt denken sich die Studierenden selbst Funktionen aus und überlegen sich, ob diese (2) erfüllen oder nicht.
Beispielsweise sieht man für $f(x)=\sin (x)$, dass die Aussage mit $\varepsilon=3$ gilt. Im Falle $f(x)=2 x$ gilt die Aussage nicht, da zu vorgegebenem $\varepsilon>0$ die Implikation für $(x, \delta, y)=(0,2 \varepsilon, \varepsilon)$ verletzt ist.

- Erfüllt jede stetige Funktion (2)? Gibt es unstetige Funktionen, die (2) erfüllen?

Wir haben bereits gesehen, dass (2) für die stetige Funktion $f(x)=2 x$ nicht gilt. Umgekehrt gilt (2) mit $\varepsilon=2$ für die charakteristische Funktion einer beliebigen Teilmenge von $\mathbb{R}$, die in der Regel unstetig ist.

- Wie lässt sich (2) veranschaulichen? Wie sieht der Graph einer Funktion $f: \mathbb{R} \rightarrow \mathbb{R}$ aus, die (2) erfüllt?

Die Studierenden erstellen passend zu (2) eine Reihe kleiner Skizzen. Durch die Abfolge der Skizzen - oder alternativ das sukzessive Erstellen einer Skizze - wird die Bedeutung der Reihenfolge in (2) hervorgehoben.

In der folgenden Ausführung betrachten wir (2) als geltende Voraussetzung und wollen daraus Eigenschaften für $f$ folgern. Dies spiegelt sich im verwendeten Sprachgebrauch wider.

(A) $\exists \varepsilon>0$ : Uns wird eine Länge $\varepsilon>0$ gegeben (in vertikaler Richtung).

(B) $\forall x \in \mathbb{R}$ : Wir dürfen uns einen beliebigen Punkt $x \in \mathbb{R}$ aussuchen (auf der $x$-Achse). Daraus ergibt sich ein Funktionswert $f(x)$.

(C) $\forall \delta>0$ : Wir dürfen uns eine beliebige Länge $\delta>0$ aussuchen (horizontal).

(D) $\forall y \in \mathbb{R}:|x-y|<\delta \Longrightarrow|f(x)-f(y)|<\varepsilon$ : Wir dürfen uns einen weiteren Punkt $y \in \mathbb{R}$ beliebig aussuchen (auf der $x$-Achse). Ist dessen Abstand zu $x$ größer oder gleich $\delta$, so ist die Implikation automatisch erfüllt und es ergibt sich keine interessante Folgerung. Ist der Abstand jedoch kleiner als $\delta$, dann folgt aus (2), dass $f(y)$ von $f(x)$ weniger als $\varepsilon$ entfernt ist.

Entscheidend für das Verständnis von (2) ist natürlich, an welche Quantoren die einzelnen Variablen gebunden sind. Die Konsequenzen davon lassen sich anhand der Skizzen auf S. 66 weiter erkunden oder in weiteren Skizzen darstellen. So könnte man von hinten beginnend zunächst überlegen, dass man für $y$ jeden Punkt im Bereich $(x-\delta, x+\delta)$ einsetzen kann und folglich der Graph von $f$ über diesem Bereich in einer im Punkt $(x, f(x))$ zentrierten Box der Breite $2 \delta$ und Höhe $2 \varepsilon$ liegen muss. AnschlieBend kann man weiter feststellen, dass man $\delta$ beliebig groß wählen kann und somit der gesamte Graph von $f$ in einem horizontal unendlich ausgedehnten und vertikal um $f(x)$ zentrierten Streifen der Höhe $2 \varepsilon$ liegt.

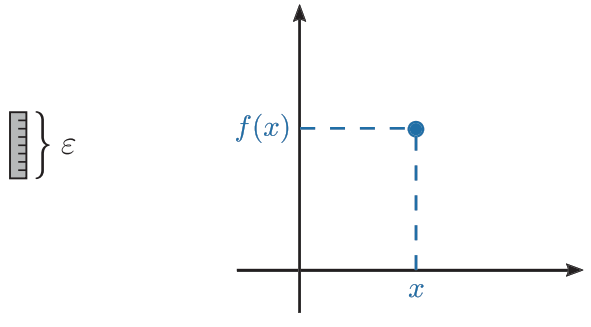

(A)
(B)

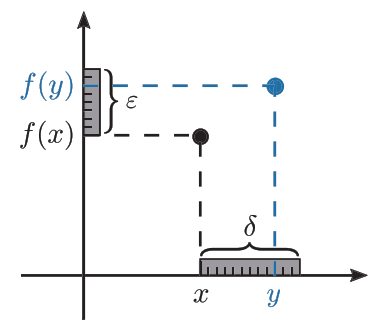

(C)

(D) 

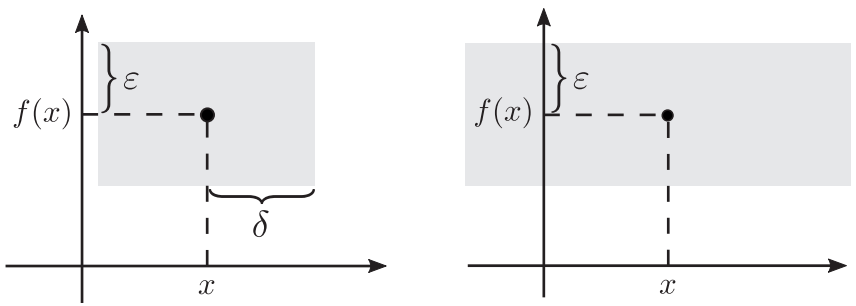

- Wie lässt sich (2) in Worten beschreiben? Kann man (2) auf einfachere, aber äquivalente Art und Weise ausdrücken? Anhand der vorherigen Überlegungen könnten die Studierenden zunächst die Vermutung äußern, dass eine Funktion, die (2) erfüllt, beschränkt sein muss, oder auch, dass (2) sogar äquivalent zur Beschränktheit ist. Im nächsten Schritt kann man die Studierenden dann auffordern, eine formale Definition von Beschränktheit zu geben, etwa

$$
\exists C>0: \forall x \in \mathbb{R}:|f(x)| \leq C,
$$

und deren Äquivalenz zu (2) zu beweisen oder eben doch ein Gegenbeispiel zu finden. Im vorliegenden Fall ist der Beweis der Äquivalenz (für erfahrene Mathematiker) leicht möglich und für Studienanfänger eine gute Übung sowohl für das Nachweisen wie auch das Anwenden quantifzierter Aussagen.

Beweis von,$(2) \Longleftrightarrow(3)^{\prime \prime}$. Sei $f: \mathbb{R} \rightarrow \mathbb{R}$ eine Funktion.

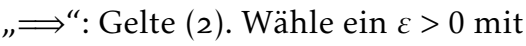

$$
\begin{aligned}
& \forall x \in \mathbb{R}: \forall \delta>0: \forall y \in \mathbb{R}: \\
& \qquad|x-y|<\delta \Longrightarrow|f(x)-f(y)|<\varepsilon .
\end{aligned}
$$

Zum Nachweis von (3) setzen wir $C:=\varepsilon+|f(0)|$. Sei $x \in \mathbb{R}$ gegeben. Wir wenden nun $(*)$ mit diesem $x, \delta:=|x|+1$ und $y:=0$ an. Wegen $|x-y|=|x|<|x|+1=\delta$ erhalten wir $|f(x)-f(0)|<\varepsilon$. Mittels Dreiecksungleichung folgt

$$
\begin{aligned}
|f(x)|=|f(x)-f(0)+f(0)| & \leq|f(x)-f(0)|+|f(0)| \\
& \leq \varepsilon+|f(0)|=C .
\end{aligned}
$$

Damit ist (3) nachgewiesen.

„“: Gelte (3). Wähle ein $C>0$ mit $|f(x)| \leq C$ für alle $x \in \mathbb{R}$. Zum Nachweis von (2) setzen wir $\varepsilon:=2 C+1$. Seien $x \in \mathbb{R}, \delta>0$ und $y \in \mathbb{R}$ mit $|x-y|<\delta$ gegeben. Nach Wahl von $C$ gelten $|f(x)| \leq C$ und $|f(y)| \leq C$. Mittels Dreiecksungleichung folgt nun

$$
|f(x)-f(y)| \leq|f(x)|+|f(y)| \leq C+C<2 C+1=\varepsilon .
$$

Damit ist (2) nachgewiesen.

- Was ist, wenn man in (2) die Reihenfolge der Quantoren (als relative Quantoren samt $\varepsilon>0, x \in \mathbb{R}, \ldots$ ) vertauscht oder einen oder mehrere der Quantoren durch den jeweils anderen ersetzt? Wann ergeben sich äquivalente, stärkere oder schwächere Aussagen?

Im Gegensatz zu einem völlig neuen Würfeln kann so nochmals speziell die Wichtigkeit der Reihenfolge bzw. der Wahl eines Quantors betont werden. Als Lehrperson kann man ganz gezielt gewisse Abänderungen vorschlagen, so würde hier zum Beispiel das Verrücken von $\exists \varepsilon>0$ an die dritte Stelle zur Bedingung der lokalen Beschränktheit führen.

Weitere Beispiele gewürfelter Aussagen

Zweites Beispiel

Hat man mit $\bullet \because \because \because$ die Aussage

$$
\begin{aligned}
\forall \varepsilon>0: \forall x \in \mathbb{R}: \exists \delta>0: & \forall y \in \mathbb{R}: \\
& |x+y|<\delta \Longrightarrow|f(x)-f(y)|<\varepsilon
\end{aligned}
$$

erwürfelt, so könnte Folgendes passieren: Zunächst bemerkt man, dass man die Reihenfolge der ersten beiden Allquantoren vertauschen darf und dass

$$
\begin{aligned}
\forall x \in \mathbb{R}: \forall \varepsilon>0: \exists \delta>0: & \forall y \in \mathbb{R}: \\
& |x+y|<\delta \Longrightarrow|f(x)-f(y)|<\varepsilon
\end{aligned}
$$

bis auf das Vorzeichen bei $x+y$ die $\varepsilon$ - $\delta$-Definition von Stetigkeit ist. Wählt man darin speziell stets $y=-x$, so gilt jeweils $|x+y|=0$. Somit braucht man den Quantor mit $\delta$ nicht mehr und erhält als Folgerung

$$
\forall x \in \mathbb{R}: \forall \varepsilon>0: \quad|f(x)-f(-x)|<\varepsilon .
$$

Da $\varepsilon>0$ beliebig ist, muss $f$ eine gerade Funktion sein, also $f(x)=f(-x)$ für alle $x \in \mathbb{R}$ erfüllen. Mit dieser Erkenntnis können wir in der ursprünglichen Aussage überall $y$ durch $-y$ ersetzen. Beim Allquantor ist keine Änderung nötig, aus $x+y$ wird $x-y$ und $f(x)-f(y)$ bleibt unverändert, weil $f$ eine gerade Funktion ist. Wir erhalten also

$$
\begin{aligned}
\forall x \in \mathbb{R}: \forall \varepsilon>0: \exists \delta>0: & \forall y \in \mathbb{R}: \\
& |x-y|<\delta \Longrightarrow|f(x)-f(y)|<\varepsilon
\end{aligned}
$$

und erkennen, dass $f$ stetig sein muss. Insgesamt haben wir also gesehen, dass aus der gewürfelten Bedingung folgt, dass $f$ gerade und stetig ist. Es auch nicht kompliziert, einzusehen, dass dies sogar eine Äquivalenz ist.

\section{Drittes Beispiel}

Bei $\bullet \because \because$, also

$$
\begin{aligned}
\forall \varepsilon>0: \forall x \in \mathbb{R}: \exists \delta & >0: \forall y \in \mathbb{R}: \\
& \min \{|x|,|y|\}>\delta \Longrightarrow|f(x)-f(y)|<\varepsilon
\end{aligned}
$$

können wir

$$
\delta= \begin{cases}2|x| & \text { für } x \neq 0, \\ 1 & \text { für } x=0\end{cases}
$$

wählen, womit $\min \{|x|,|y|\}>\delta$ stets verletzt ist. Die gewürfelte Aussage gilt somit für beliebige Funktionen. 
Viertes Beispiel

Erwürfeln wir uns mit $\bullet \because: \because$ die Aussage

$$
\begin{aligned}
\forall \varepsilon>0: \exists \delta>0: \forall x & \in \mathbb{R}: \forall y \in \mathbb{R}: \\
& \min \{|x|,|y|\}>\delta \Longrightarrow|f(x)-f(y)|<\varepsilon,
\end{aligned}
$$

so beobachten wir zunächst, dass die Einschränkung auf kleine $\varepsilon>0$ und große $\delta>0$ die dazu äquivalente Aussage

$$
\begin{aligned}
\forall \varepsilon \in(0,1): \exists \delta \geq 1: & \forall x \in \mathbb{R}: \forall y \in \mathbb{R}: \\
& \min \{|x|,|y|\}>\delta \Longrightarrow|f(x)-f(y)|<\varepsilon
\end{aligned}
$$

liefert. Nach kurzem Überlegen sehen wir, dass dies äquivalent dazu ist, dass die beiden Grenzwerte $\lim _{x \rightarrow \infty} f(x)$ und $\lim _{x \rightarrow-\infty} f(x)$ existieren und übereinstimmen.

$$
\begin{aligned}
& \text { Fünftes Beispiel } \\
& \text { Bei } \because \because \because \text {, also } \\
& \qquad \varepsilon>0: \forall \delta>0: \exists x \in \mathbb{R}: \forall y \in \mathbb{R}: \\
& \qquad|x-y|+\frac{1}{1+|x|}<\delta \Longrightarrow|f(x)-f(y)|<\varepsilon
\end{aligned}
$$

erkennt man, dass die Prämisse für kleine $\delta>0$ nur wahr sein kann, wenn $|x-y|$ klein und $|x|$ groß ist. Für diese Aussage haben wir keine schöne äquivalente Umschreibung. Dafür bietet es sich nun an, wilde Funktionen zu suchen, die diese Bedingung noch erfüllen. Beispiele dafür sind alle Funktionen $f: \mathbb{R} \rightarrow \mathbb{R}$ mit

$$
|f(x)-\log x| \leq 1 / x
$$

für $x \geq 1$.

\section{Weitere mögliche Fragen für Lehrveranstaltungen}

In der folgenden Liste haben wir einige weitere mögliche Fragen gesammelt, die man Studierenden stellen kann.

- Wie kann man eine gegebene Funktion, die eine gewürfelte Aussage erfüllt, so abändern, dass die Aussage weiterhin gültig bleibt?

Man könnte hier, ausgehend von einfachen Funktionen wie etwa Polynomen oder rationalen Funktionen, reichhaltigere Beispiele konstruieren.

- Wie wild kann eine Funktion bzw. ihr Graph aussehen, die die gewürfelte Aussage noch erfüllt?

- Welche anderen Aussagen sind hinreichend oder notwendig für das Gelten der gewürfelten Aussage? Welche Gegenbeispiele zeigen, dass keine Äquivalenz vorliegt?

Falls hierbei Vermutungen geäußert werden, die sich als falsch herausstellen, kann man sich fragen, wie man die gewürfelte Aussage abändern müsste, sodass sie zu der geäußerten Vermutung passt.

- Wie lautet die Verneinung der Aussage? Ist diese leichter zu verstehen?

- Ändert es die Aussage, wenn man $\varepsilon>0$ durch $\varepsilon \in(0,1)$ oder $\varepsilon \geq 1$ ersetzt?

Kommentare

- Trotz des Namens Stetigkeitswürfel müssen die erwürfelten Aussagen nichts mit Stetigkeit zu tun haben, sie erinnern jedoch an die $\varepsilon$ - $\delta$-Definition von Stetigkeit.

○ Mit den Stetigkeitswürfeln kann man nicht nur das formale Arbeiten, sondern auch die mathematische Intuition trainieren.

- Da die erwürfelten Aussagen häufiger zumindest für Anfänger nicht leicht $\mathrm{zu}$ verstehen sind, sollte eine mathematisch erfahrene Person mitspielen. Sie kann zum Beispiel vorgeben, bei welchen Aussagen nur Beispiele oder Gegenbeispiele gesucht werden sollen und bei welchen sich eine äquivalente Beschreibung anbietet.

- Es empfiehlt sich ein allmähliches Herantasten an die erwürfelten Aussagen. Dazu eignen sich die obigen Fragestellungen.

- Beim Einsatz in Veranstaltungen im ersten Studienjahr muss man sich für jede erwürfelte Aussage viel Zeit lassen.

- Dank jahrelanger Gewöhnung sind wir Dozenten mit der Stetigkeitsdefinition vertraut, während uns eine ähnliche erwürfelte Aussage häufig kompliziert erscheint. Anfängern mag beides gleich schwer fallen. Wir können nun beim Spielen mit den Stetigkeitswürfeln die Schwierigkeiten mit derartigen Aussagen für uns neu erfahren und so mehr Verständnis für die Schwierigkeiten der Studienanfänger bekommen.

- Eine Kombination mit Funktionen auf Spielkarten, für die jeweils die Gültigkeit der erwürfelten Aussage geprüft werden soll, ist in Planung.

\section{Danksagung}

Zunächst möchten wir den Teilnehmern der EmA dafür danken, dass wir mit ihnen diese Würfel ausprobieren durften. Weiterhin danken wir allen Studierenden, die uns durch kreativ durcheinandergebrachte Definitionen, beispielsweise zur Stetigkeit, auf Gedanken gebracht haben, die letztlich zu diesen Würfeln geführt haben.

\section{Literatur}

[1] Jan-Hendrik Treude. Die Einführung in das mathematische Arbeiten an der Universität Konstanz. In Vorbereitung.

Prof. Dr. Oliver Schnürer und Dr. Jan-Hendrik Treude, Universität Konstanz,

Fachbereich Mathematik und Statistik, 78457 Konstanz oliver.schnuerer@uni-konstanz.de

jan-hendrik.treude@uni-konstanz.de

Oliver Schnürer forscht und lehrt an der Universität Konstanz und hat dort die Veranstaltung Einführung in das mathematische Arbeiten ins Leben gerufen. Jan-Hendrik Treude ist als Mitarbeiter für die Einführung in das mathematische Arbeiten und individuelle zusätzliche Betreuung der Studienanfänger zuständig und darüber hinaus in der Lehre aktiv. 